\title{
Combination Treatment with Antibiotics and Glucocorticosteroids for Severe Ischemic Colitis
}

\author{
Wolfgang Kruis Michael Schiff \\ Evangelisches Krankenhaus Kalk, Klinik für Gastroenterologie, Pulmologie und Allgemeine Innere Medizin, Teaching \\ Hospital of the University of Cologne, Cologne, Germany
}

\section{Keywords}

Ischemic colitis · Conservative management ·

Prednisolone $\cdot$ Antibiotics

\begin{abstract}
Background/Aims: Ischemic colitis (IC) is most common in the elderly and patients with multiple comorbidities. It carries significant mortality. As yet no evidence-based therapeutic management exists. Aim of the study was to test therapeutic efficacy of a combination of prednisolone and antibiotics.Methods:Prospectivecohortstudy with retrospective analysis performed in a single teaching hospital in Germany. Consecutive patients with strict diagnostic criteria of severe IC, including colonoscopy, histology, and laboratory tests, were recruited. Main outcome measures were in-hospital mortality and number of operations counted within the hospital stay. Severity scores were calculated and biomarkers determined during the course of the hospital stay. Results: A total of 342 patients with an International Classification of Diseases of IC were identified. About 151 patients met the diagnostic criteria and a total of 44 patients fulfilled all inclusion and exclusion criteria of severe IC and constituted the group of patients eligible for analysis. Five out of 44 patients (11.4\%) died (in-hospital mortality). Surgery was performed in 3 patients $(6.8 \%), 2$ patients survived. The hospital stay lasted $14.0 \pm 8.5$ day and was significantly correlated with
\end{abstract}

comorbidity $\left(r_{\mathrm{s}}=0.314, p=0.038\right)$. No serious adverse events were observed. Conclusion: This is the first prospective study on therapeutic efficacy and safety in severe IC. The combination of intravenous antibiotics and intravenous prednisolone turned out to be safe and revealed promising efficacy.

(c) 2019 S. Karger AG, Basel

\section{Introduction}

Ischemic colitis (IC) is a condition of inadequate perfusion leading to colonic inflammation [1]. Typical symptoms are hematochezia, abdominal pain, and diarrhea. The severity varies widely. Over three-fourths of cases are the milder, nongangrenous form, but IC can progress to necrosis with a mortality of reaching 50\% [2]. The same review describes an overall incidence of poor prognosis of $22 \%$ in patients with IC. The mortality in unselected cases varied from 3.7 to $32.6 \%$, and surgical interventions were performed in 3.9-79.1\% of the patients [2]. IC ranks second among nonmalignant gastrointestinal disease causes of in-hospital mortality [3]. In this context, it is to note that a population-based study from Olmsted county, USA, describes increasing incidence of IC of 22/100,000 between 2005 and 2009. In-hospital mortality was $11.5 \%$ and rates of surgery were $17.0 \%$ [4].

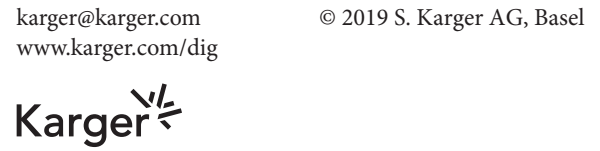

Prof. Dr. Wolfgang Kruis 
IC is usually managed conservatively with surgery reserved as rescue therapy. American College of Gastroenterology states in their clinical guidelines: "Antimicrobial therapy should be considered for patients with moderate or severe disease (strong recommendation, very low level of evidence)" [5].

The most recent review of IC management [6] quotes 6 observational studies with small case series. In 5 of these studies, treatment was variable being determined at the discretion of the attending physician. One study describes therapy with rebamipide enemas in 6 patients with moderate IC [7]. Rebamipide, an antiulcer drug, affects radicals and mucin. The concept of anti-inflammatory antiulcer therapy in severe IC holds reasonable considerations. In 2 large, unselected populations of patients with IC inflammation followed by ulceration of the colonic mucosa was the most common pathological finding $[8$, 9]. Corticosteroids are well established for the treatment of inflammatory and ulcerative bowel diseases but have never been reported in IC.

Thus, several reasons gave cause for our study: the lack of any formal studies on treatment, especially in selected patients with severe IC and bad prognosis, the lack of therapeutic concepts, and missing experience of effects and safety of anti-inflammatory therapy with steroids. Because of the unclear situation, it seems to be justified to perform as a first step an open observational cohort study following a strictly defined protocol. The results may build up the basis for further controlled studies.

\section{Materials and Methods}

\section{Study Design}

This is a prospective cohort study with retrospective analysis. In the absence of evidence-based management protocols or recommendations from consensus conferences, we created for our department standard operating procedures (SOPs) for the diagnosis and treatment of IC. Since 2003 patients were managed in our unit along with SOPs. SOPs are consented by the Ethical Committee and the department of quality and risk management of the hospital.

\section{Study Population and Assessments}

Searching for patients with International Classification of Diseases K55.0, K55.1, and K55.9, we found 342 patients hospitalized in the Evangelisches Krankenhaus Kalk between 2003 and 2012 (Fig. 1). All patients had given informed consent.

Inclusion criteria for the final analysis were need of hospitalization, at least 1 day of predefined treatment, complete documentation of all clinical parameters including Charlson comorbidity index [10], total colonoscopy with severity index [11], positive histology, and laboratory tests (full blood count, C-reactive protein [CRP], lac-

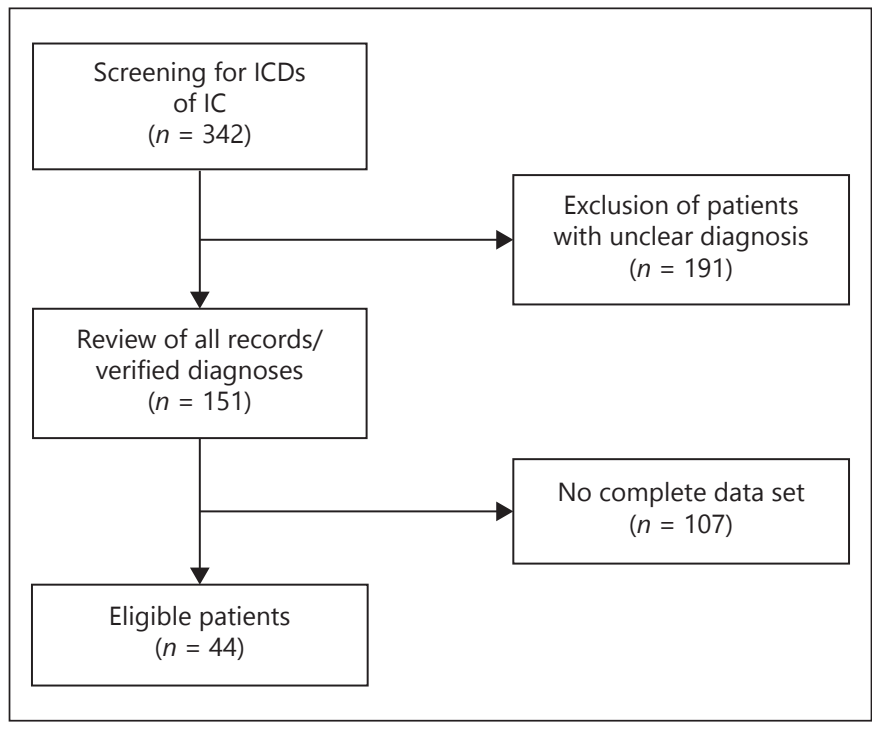

Fig. 1. CONSORT diagram of the patients in the study. ICD, International Classification of Diseases; IC, ischemic colitis.

tate dehydrogenase [LDH], and total serum protein). Also stool testing for pathogens including Clostridium difficile was required. Exclusion criteria were known other organic intestinal diseases such as inflammatory bowel disease (IBD), colonic malignancy, or diverticulitis. Also excluded were patients with a different source for intestinal bleeding (e.g., upper GI-bleeding, angiodysplasia).

Baseline severity of IC was retrospectively calculated according to a validated prognostic scoring model [12]. The model processes 3 parameters, presence of ulcers as seen by colonoscopy, shock, and tachycardia, to a statistical grading of 8 groups from group 1 (very mild) to group 8 (very severe). Taken together, for groups $5-8$, a specificity of $88.7 \%$ for severe IC was calculated, while patients in groups $6-8$ showed a specificity of $93.2 \%$.

\section{Treatment}

After the diagnosis was established, the treatment followed SOPs, comprising in-hospital care, general measures such as nothing by mouth, intravenous fluids with electrolytes if needed, and subcutaneous heparin in preventative doses. Specific therapy included i.v. prednisolone (50-75 mg/day) and combined i.v. antibiotics, metronidazole (500 $\mathrm{mg}$ bid), and mezlocillin (2 g tid). In 2009, mezlocillin was stopped by the manufacturer and was replaced in the standard regimen by piperacillin/tazobactam ( $4 / 0.5 \mathrm{~g}$ tid). As soon as possible, intravenous therapy was switched to oral treatment.

\section{Objectives}

Primary endpoints were in-hospital mortality and need of surgery. Secondary endpoints were safety, length of hospital stay, and time to decreasing the dose of systemic steroids.

\section{Statistical Analysis}

Data are presented as mean \pm SD. Normal distribution was tested by the Kolmogorov-Smirnov test. On this basis, $t$ test was performed for differences. Bivariate correlation according to Pearson was calculated. SPSS for Macintosh version 22.0 was used for all 
Table 1. Biographic and clinical data of patients with IC $(n=44)$

\begin{tabular}{ll}
\hline & $n(\%)$ \\
\hline Age, years, mean \pm SD & $73 \pm 13$ \\
Gender, male & $26(59.1)$ \\
Comorbidity $($ Charlson Index $>3)$ & $23(52.3)$ \\
LDH (> ULN, 225 U/L) & $14(31.8)$ \\
Leucocyte count $\left(>\mathrm{ULN}, 10.0 \times 10^{9}\right)$ & $27(61.4)$ \\
Serum sodium $(<\mathrm{LLN},<136 \mathrm{mmol} / \mathrm{L})$ & $19(43.2)$ \\
Systolic blood pressure $(<90 \mathrm{~mm} \mathrm{Hg})$ & $6(13.6)$ \\
Tachycardia $(>90 /$ min $)$ & $30(68.2)^{*}$ \\
Signs of peritonitis (tension, free liqids) & $11(25.0)$ \\
\hline
\end{tabular}

* Out of 43 patients (1 patient missing data).

IC, ischemic colitis; LDH, lactate dehydrogenase.

Table 2. Endoscopic findings in 44 patients with IC

\begin{tabular}{lcc}
\hline Frequency & Proportion, \% & Patients, $n$ \\
\hline Length of colitis, cm & & \\
$\quad<10$ & 6 & 13.6 \\
$\quad>10$ & 38 & 86.4 \\
Endoscopic severity* & 6 & \\
$\quad$ Mild & 38 & 13.6 \\
$\quad$ Severe & & \\
Localisation & 6 & 13.6 \\
$\quad$ Ascending colon & 3 & 6.8 \\
$\quad$ Transverse colon & 3 & 43.8 \\
$\quad$ Descending/sigmoid colon & 16 & 36.3 \\
$\quad \geq 2$ colonic segments & & \\
\hline
\end{tabular}

* According to Beppu et al. [11].

IC, ischemic colitis.

analyses. Statistics were performed under the supervision of the Institut für Medizinische Statistik und Epidemiologie of the university of Cologne by Dipl. math. Ingrid Becker.

\section{Results}

\section{Patients}

The electronic search system of the hospital identified a total of 342 patients with International Classification of Diseases K55 between 2003 and 2012. One hundred and fifty-one patients achieved strict diagnostic criteria and a total of 44 patients fulfilled all inclusion and exclusion criteria, thus constituting the group of patients eligible for analysis.

As expected, the patient population was older, $72.6 \pm$ 13.5 years (mean $\pm \mathrm{SD}$ ), but in contrast to the literature, there was a preponderance of male patients (59.1\%). A Charlson comorbidity index of $3.7 \pm 2.7$ demonstrates the severity of the very ill patients. Charlson index is a weighted score consisting of 5 levels that take into account the number and the seriousness of comorbid disease, for example, a score of $\geq 3$ has shown a 1 year mortality $>50 \%$ [10]. Biographic and clinical data of all patients are listed in Table 1.

\section{Endoscopy}

In addition, endoscopy depicted severe mucosal lesions (Table 2). Most patients showed a long segment $(>10 \mathrm{~cm})$ ischemic reaction. In total, $86.7 \%$ of the patients had longitudinal or circumferential ulcers which are endoscopically severe IC according to the classification by Beppu et al. [11].

The retrospectively calculated prognostic severity score of Chung et al. [12] attributed 6 patients to group 2, 7 patients to group 4, 24 patients to group 6 , and 6 patients to group 8 ( $n=43$ patients with 1 patient missing data). Thus, assessing 30 out of $43(69.7 \%)$ patients to a risk score of severe IC and $7 / 43$ patients (16.2\%) to a risk score of moderate severity.

\section{Biomarker}

The most sensitive marker was CRP. Forty out of 44 patients had elevated blood levels $(>0.5 \mathrm{mg} / \mathrm{dL})$. The total median was $7.9 \mathrm{mg} / \mathrm{dL}$ with a wide range $(0.1-40.8 \mathrm{mg} /$ $\mathrm{dL})$. Most likely because of this range, the difference of CRP between severe IC (mean \pm SD; $8.4 \pm 10.0 \mathrm{mg} / \mathrm{dL}$ ) and mild IC $(5.2 \pm 8.3 \mathrm{mg} / \mathrm{dL})$ did not achieve statistical significance $(p=0.46)$.

Other biomarkers showed not only less pronounced alterations but also wide variations such as leucocytes $\left(12.1 \pm 5.0 \times 10^{9} / \mathrm{L}\right.$; normal range $\left.4-10 \times 10^{9} / \mathrm{L}\right), \mathrm{LDH}$ $(259.31 \pm 32.9 \mathrm{U} / \mathrm{L}$; normal range: $<225 \mathrm{U} / \mathrm{L})$, and total protein $(5.8 \pm 1.3 \mathrm{~g} / \mathrm{dL}$; normal range: $>6.6 \mathrm{~g} / \mathrm{dL})$. No significant differences between patients with mild and severe IC could be seen.

\section{Primary Outcome}

Five patients (3 males, 62-93 years of age) out of 44 patients (11.4\%) died (in-hospital mortality). One of these 5 patients had an emergency operation because of free perforation. All patients presented with severe lower gastrointestinal bleeding in need of multiple blood transfusions. All patients had considerable comorbidity such as general atherosclerosis also including abdominal vessels and coronary heart disease and in 1 case advanced bronchial carcinoma. Three patients died from pulmonary sepsis, one from renal failure (superimposed to 


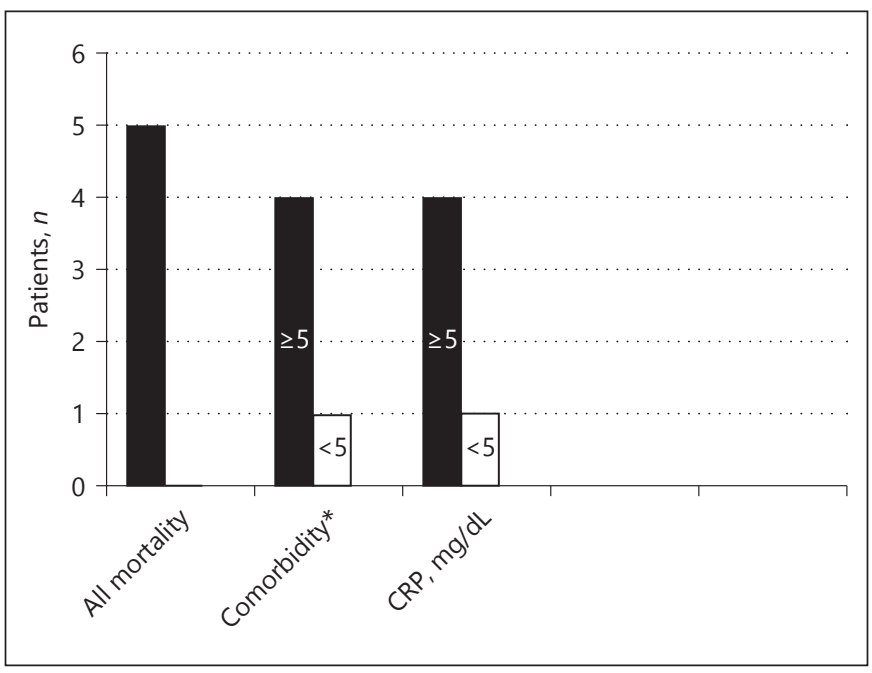

Fig. 2. Mortality out of 44 patients with IC according to comorbidity and C-reactive protein (CRP). * Charlson Index. CRP, C-reactive protein

chronic kidney disease), and the 93-year-old patient died from cardiovascular failure. Surgery was performed in 3 patients (6.8\%), 2 patients survived.

All patients with fatal outcome of their disease had severe endoscopic findings. Endoscopic length of the diseased segment and localization showed no relationship to outcomes.

\section{Secondary Outcomes}

Comorbidity had a strong influence on the course of IC. The Charlson index was $3.2 \pm 2.5$ in the patient group without mortality or need for surgery while it was significantly ( $p=0.003$ ) higher $6.6 \pm 2.3$ in the group with mortality and/or surgery. Likewise, patients with mortality and/or surgery had a $>$ two-fold Charlson comorbidity index ( $p=0.002$; Fig. 2).

Among various laboratory tests (Table 2), mortality was associated to higher initial CRP serum concentrations $(p=0.05)$. Patients with initial CRP higher than $10 \mathrm{mg} / \mathrm{dL}$ exhibited a 13-fold increased risk for a fatal outcome (OR 13.3 [95\% CI 1.32-134.93]; $p=0.02$; Fig. 1).

As a response to treatment, CRP serum concentration dropped significantly within the hospital stay $(p<0.001)$. In patients with severe IC, CRP decreased from initially 8.4 to $1.9 \mathrm{mg} / \mathrm{dL}$, while this difference was in mild IC 5.2 to $1.3 \mathrm{mg} / \mathrm{dL}$. Other markers such as leucocytes, $\mathrm{LDH}$, and total protein showed no relevant changes.

The hospital stay was $14.0 \pm 8.5$ days and was significantly correlated to comorbidity $\left(r_{\mathrm{s}}=0.31, p=0.04\right)$. Weaning from systemic steroids was performed accord- ing to clinical and laboratory (CRP) improvements and started after $6.8 \pm 4.2$ days. Here, a significant correlation between length of the endoscopic inflammation $(</>10 \mathrm{~cm})$ and time to the switch from intravenous to oral treatment existed $(r=0.33, p=0.03)$.

\section{Safety Assessments}

In this group of multimorbid and severely ill patients adverse events and tolerability of the treatment are hardly to interpret. As reported, no unexpected side effects such as allergic reaction, toxic colon, or new Clostridium difficile infection were identified.

\section{Discussion}

This study is the first to describe results of predefined conservative management in a cohort of patients with colonoscopy and histology proven severe IC. All patients received prednisolone and antibiotics. Because of a lack of any other trials, it is awkward to assess therapeutic effects of this uncontrolled study. Although, it is clear that outcomes of IC depend on the severity of the disease, reviews describe results mostly in unselected patients, thus hampering direct comparisons.

Several risk factors for severe IC and bad prognosis have been described $[2,5]$. Certain endoscopic findings have been related to severity of IC [11]. Even a validated prognostic scoring model has been developed [12]. According to those criteria, our study population reflects certainly a group of patients with severe IC and bad prognosis.

Few studies on mortality and surgical interventions consider distinct factors for severity. A study defining old age as predictive for bad outcomes investigated patients with a mean age of $79.9 \pm 10.2$ years compared to $72.6 \pm$ 13.5 years of our population and found a mortality rate of $19.5 \%$ [13]. In a group of 47 patients with nonocclusive colonic ischemia mortality of $>20 \%$ was reported [14]. A subgroup of patients with right-sided ischemic reaction and a Charlson index of comorbidity of $2.3 \pm 2.1$ was related to a mortality rate of $24.6 \%$ [15]. To note, here we report patients with a much worse Charlson index of $3.7 \pm 2.7$, which underlines again the bad prognosis of our study population. A more recent paper described in a selected group of patients with severe IC $(n=33)$ a mortality of $84.8 \%$ and a rate of operation of $15.1 \%$. Unfortunately, definition of severe IC and times of observation were not clearly defined [16]. To summarize, rates of inhospital mortality (11.4\%) and surgical interventions 
$(6.8 \%)$ in our study population with proven severe IC compare favorably to results from the literature.

While rigorous evidence is lacking, the American College of Gastroenterology clinical guidelines recommend for conservative management of IC fasting, intravenous fluids, and correction of underlying conditions [5]. In addition, there is a wide consensus by expert opinions on the use of broad-spectrum antibiotic coverage. In severe disease, surgery should be considered [5]. Glucocorticosteroids are explicitly not recommended, though firm reasons against their use are not given. A single experiment in rats found significantly greater areas of colonic ischemic changes with steroids than in the control group which led to the suggestion that systemic administration of immunosuppressive agents may augment the development of IC [17]. Thus, the question arises as how does the concept of combination therapy with antibiotics and glucocorticoids relate to the pathophysiology of IC.

As yet the pathogenesis of IC is not completely understood. It is thought that local hypoperfusion and subsequent reperfusion cause mucosal injury and dysfunction allowing bacterial translocation through the intestinal mucosa. Although antibiotic treatment of IC is often mentioned, clinical evidence from therapeutic studies with antibiotics is limited because of a lack of prospective controlled series or even studies with prospective therapy [5]. In a mouse model of IC, commensal bacteria influenced mucosal integrity. Depletion of the microbiota decreased the expression of Toll-like receptors (TLR2 and TLR4), leading to the reduction of inflammatory mediators, such as TNFa, IL-6, and cyclogenase-2 [18]. Postischemic colonic injury involves an inflammatory cascade in intestinal tissues through the recruitment of circulating inflammatory cells. Resident myocytes initiate inflammation through TNFa production in postischemic conditions [19]. Those findings show similarity to the pathophysiology of IBD. Interestingly, typical IBD-immune markers such as perinuclear anti-neutrophil cytoplasmic antibodies and anti-Saccharomyces cervisiae antibodies were found in sera of patients with recurrent and chronic IC [20]. Glucocorticosteroids are a well-established therapeutic principle in IBD. Thus, most likely, pathogenesis of IC is multifactorial including crucial steps such as disruption of mucosal integrity, bacterial translocation, and initiation of local immune reactions.

The current regimen was well tolerated. There were no allergic reactions to antibiotics and no episodes of antibiotic-associated diarrhea were reported. Prednisolone was given only short term that presumably prevented typical long-term adverse events. Severe events such as develop- ment of toxic colon or sepsis were not observed. Patients who died or were in need of surgery during the treatment with prednisolone were characterized by prognostic very bad criteria at baseline including severe endoscopic findings, considerable comorbidity, and high CRP levels. No fatal outcomes were attributable to the use of prednisolone.

Our work has several limitations. Though the patients were treated prospectively according to a defined protocol, analyses were retrospective. It is a case series without control group. The number of patients meeting all protocol criteria was relatively small. For reasons of availability, there was a change in antibiotics during the study. The strength of the study is strict diagnosis and characterization of disease severity including total colonoscopy and histologic analysis. It is the first formal study ever in severe IC. The basis for the choice of the study medication is based on pathophysiological considerations. The study allows assessments of the potential benefit and safety of a novel treatment regimen in a well-defined patient population.

\section{Conclusions}

This study adds new data to the as yet unsolved question of conservative management of severe IC. Combination therapy with intravenous antibiotics and intravenous prednisolone raised no safety concerns and was associated with promising therapeutic effects. This very preliminary results should stimulate further and controlled trials in the as yet disregarded field of conservative management of severe IC.

\section{Acknowledgments}

The authors are grateful to all medical personal of our hospital who have contributed with patients and documentation (study nurse: Fisnike Verlaku). Statistics were supervised by Dipl Mat. Ingrid Becker, Institut für Med. Statistik und Epidemiologie of the University of Cologne. There was no medical writer, but a native expert in writing, Elizabeth Kovach, edited the revised manuscript.

\section{Disclosure Statement}

The authors declare that they have nothing to disclose.

\section{Funding Source}

No funding. 


\section{References}

1 Higgins PD, Davis KJ, Laine L. Systematic review: the epidemiology of ischaemic colitis. Aliment Pharmacol Ther. 2004 Apr;19(7): 729-38.

2 Sun D, Wang C, Yang L, Liu M, Chen F. The predictors of the severity of ischaemic colitis: a systematic review of 2823 patients from 22 studies. Colorectal Dis. 2016 Oct;18(10):94958.

3 Peery AF, Dellon ES, Lund J, Crockett SD, McGowan CE, Bulsiewicz WJ, et al. Burden of gastrointestinal disease in the United States: 2012 update. Gastroenterology. 2012 Nov; 143(5):1179-1187.e3.

4 Yadav S, Dave M, Edakkanambeth Varayil J, Harmsen WS, Tremaine WJ, Zinsmeister AR, et al. A population-based study of incidence, risk factors, clinical spectrum, and outcomes of ischemic colitis. Clin Gastroenterol Hepatol. 2015 Apr;13(4):731-8.e1.

5 Brandt LJ, Feuerstadt P, Longstreth GF, Boley SJ; American College of Gastroenterology. ACG clinical guideline: epidemiology, risk factors, patterns of presentation, diagnosis, and management of colon ischemia (CI). Am J Gastroenterol. 2015 Jan;110(1) $18-44$.

6 O'Neill S, Yalamarthi S. Systematic review of the management of ischaemic colitis. Colorectal Dis. 2012 Nov;14(11):e751-63.

7 Matsumoto S, Tsuji K, Shirahama S. Rebamipide enema therapy for left-sided ischemic colitis patients accompanied by ulcers: open label study. World J Gastroenterol. 2008 Jul;14(25):4059-64.
8 Montoro MA, Brandt LJ, Santolaria S, Gomollon F, Sánchez Puértolas B, Vera J, et al.; Workgroup for the Study of Ischaemic Colitis of the Spanish Gastroenterological Association (GTECIE-AEG). Clinical patterns and outcomes of ischaemic colitis: results of the Working Group for the Study of Ischaemic Colitis in Spain (CIE study). Scand J Gastroenterol. 2011 Feb;46(2):23646.

9 Fenster M, Feuerstadt P, Brandt LJ, Mansoor MS, Huisman T, Aroniadis OC. Real-world multicentre experience of the pathological features of colonic ischaemia and their relationship to symptom duration, disease distribution and clinical outcome. Colorectal Dis. 2018 Dec;20(12):1132-41.

10 Charlson ME, Pompei P, Ales KL, MacKenzie CR. A new method of classifying prognostic comorbidity in longitudinal studies: development and validation. J Chronic Dis. 1987; 40(5):373-83.

11 Beppu K, Osada T, Nagahara A, Matsumoto K, Shibuya T, Sakamoto N, et al. Relationship between endoscopic findings and clinical severity in ischemic colitis. Intern Med. 2011; 50(20):2263-7.

12 Chung JW, Cheon JH, Park JJ, Jung ES, Choi $\mathrm{EH}, \mathrm{Kim} \mathrm{H}$. Development and validation of a novel prognostic scoring model for ischemic colitis. Dis Colon Rectum. 2010 Sep;53(9): 1287-94.

13 Mosele M, Cardin F, Inelmen EM, Coin A, Perissinotto E, Sergi G, et al. Ischemic colitis in the elderly: predictors of the disease and prognostic factors to negative outcome. Scand J Gastroenterol. 2010 Apr;45(4):42833.

14 Longo WE, Ballantyne GH, Gusberg RJ. Ischemic colitis: patterns and prognosis. Dis Colon Rectum. 1992 Aug;35(8):726-30.

15 Brandt LJ, Feuerstadt P, Blaszka MC. Anatomic patterns, patient characteristics, and clinical outcomes in ischemic colitis: a study of 313 cases supported by histology. Am J Gastroenterol. 2010 Oct;105(10):2245-52.

16 Choi SR, Jee SR, Song GA, Park SJ, Lee JH, Song CS, et al. Predictive factors for severe outcomes in Ischemic Colitis. Gut Liver. 2015 Nov;9(6):761-6.

17 Gomella LG, Gehrken GA, Hagihara PF, Flanigan RC. Ischemic colitis and immunosuppression. An experimental model. Dis Colon Rectum. 1986 Feb;29(2):99-101.

18 Yoshiya K, Lapchak PH, Thai TH, Kannan L, Rani P, Dalle Lucca JJ, et al. Depletion of gut commensal bacteria attenuates intestinal ischemia/reperfusion injury. Am J Physiol Gastrointest Liver Physiol. 2011 Dec;301(6): G1020-30.

19 Jarry A, Bach-Ngohou K, Masson D, Dejoie T, Lehur PA, Mosnier JF, et al. Human colonic myocytes are involved in postischemic inflammation through ADAM17-dependent TNFalpha production. Br J Pharmacol. 2006 Jan;147(1):64-72.

20 Brandt LJ, Aroniadis OC, Feuerstadt P. On the prevalence and utility of IBD-immune markers in colon ischemia. Am J Gastroenterol. 2015;110(10):1507. 